\title{
Monitoring the promoter activity of long noncoding RNAs and stem cell differentiation through knock-in of sgRNA flanked by tRNA in an intron
}

\author{
Yu-Ting Zhao ${ }^{1}$ and Yangming Wang $\mathbb{1}^{2}$
}

Dear Editor,

The majority of mammalian genome is transcribed to RNA transcripts, of which only a very small percentage code for proteins ${ }^{1}$. As a result, thousands of RNAs that do not code for proteins are produced in cells, including microRNAs (miRNAs) and long noncoding RNAs (lncRNAs). These noncoding RNAs exert regulatory functions in various physiological and pathological conditions ${ }^{2}$. In addition, numerous noncoding RNAs are expressed in a tissue- and cell-specific manner ${ }^{1}$. Thus, a reporter that faithfully reflects the expression or activity of noncoding RNAs can provide useful tools not only for uncovering the regulators of noncoding RNAs, but also for tracking cell fate and disease status. Previously we have designed a miRNA inducible CRISPR-Cas9 platform that can serve as a sensor for miRNA activities ${ }^{3}$. However, designing a reporter for long noncoding RNAs has not been easy due to its untranslatable nature and low expression level. Here, we design an sgRNA precursor in an intron (GRIT) strategy that can monitor the promoter activity of lncRNAs (Fig. 1a). Furthermore, we show that GRIT can be used to track differentiation status of stem cells.

The design of GRIT includes three key elements (Fig. 1a): dCas9-VPR expressed under the control of a constitutively active CAGGS promoter ${ }^{3}$, an RFP gene under the control of a tetracycline-inducible promoter (TRE) ${ }^{3}$, and a transfer $\mathrm{RNA}^{\mathrm{Gln}}\left(\mathrm{tRNA}^{\mathrm{Gln}}\right)^{4}$-flanked sgRNA that is integrated in an endogenous noncoding RNA locus through homologous

\footnotetext{
Correspondence: Yangming Wang (yangming.wang@pku.edu.cn)

${ }^{1}$ Academy for Advanced Interdisciplinary Studies, Peking University, Beijing,

China

${ }^{2}$ Institute of Molecular Medicine, College of Future Technology, Peking

University, Beijing, China
}

recombination. To minimize the impact of tRNA-sgRNA knock-in on lncRNAs, we chose genome region that will be expressed as an intron to knock-in tRNA-sgRNAs. In addition, for lncRNA gene without an intron, an artificially designed mini-intron-containing tRNA-sgRNA fusion sequence was knocked in. The tRNA flanking design was chosen based on our observation that tRNA-flanked sgRNA induced higher level of RFP expression when compared to ribozyme-flanked sgRNAs (Fig. 1b, c; Supplementary Fig. S1a-c and Table S1).

We then knocked the tRNA-flanked sgRNA into the second intron of Lncenc1 in mouse embryonic stem cells (ESCs) in which CAGGS-dCas9-VPR and TRE-RFP have been transgenically integrated (Fig. 1d; Supplementary Table S1). Lncenc1 is a lncRNA specifically expressed in mouse ESCs ${ }^{5}$. In ESCs with GRIT successfully integrated (Lncenc1-GRIT ESCs), we observed high level of RFP expression (Fig. 1e, f). In addition, the knock-in of tRNAsgRNA have little effect on the expression of Lncenc1 and pluripotency genes including Nanog, Oct4 (also known as Pou5f1) and Sox2 (Supplementary Fig. S2a, Tables S2 and S3). Importantly, the transcription activity of Lncenc1 locus was found not affected based on qPCR analysis of precursor RNA of Lncenc1 (Supplementary Fig. S2a).

Lncenc1 is downregulated during ESC differentiation ${ }^{5}$. To check whether GRIT can report the expression of Lncenc1 during ESC differentiation, we induced differentiation of Lncenc1-GRIT ESCs with all-trans retinoid acids (ATRA) and measured RFP expression during differentiation process. Interestingly, RFP was significantly decreased upon ATRA induced differentiation (Fig. 1g, h; Supplementary Fig. S2b). More importantly, RFP level was highly correlated to the RNA level of Lncenc1 (Supplementary Fig. S2c). 


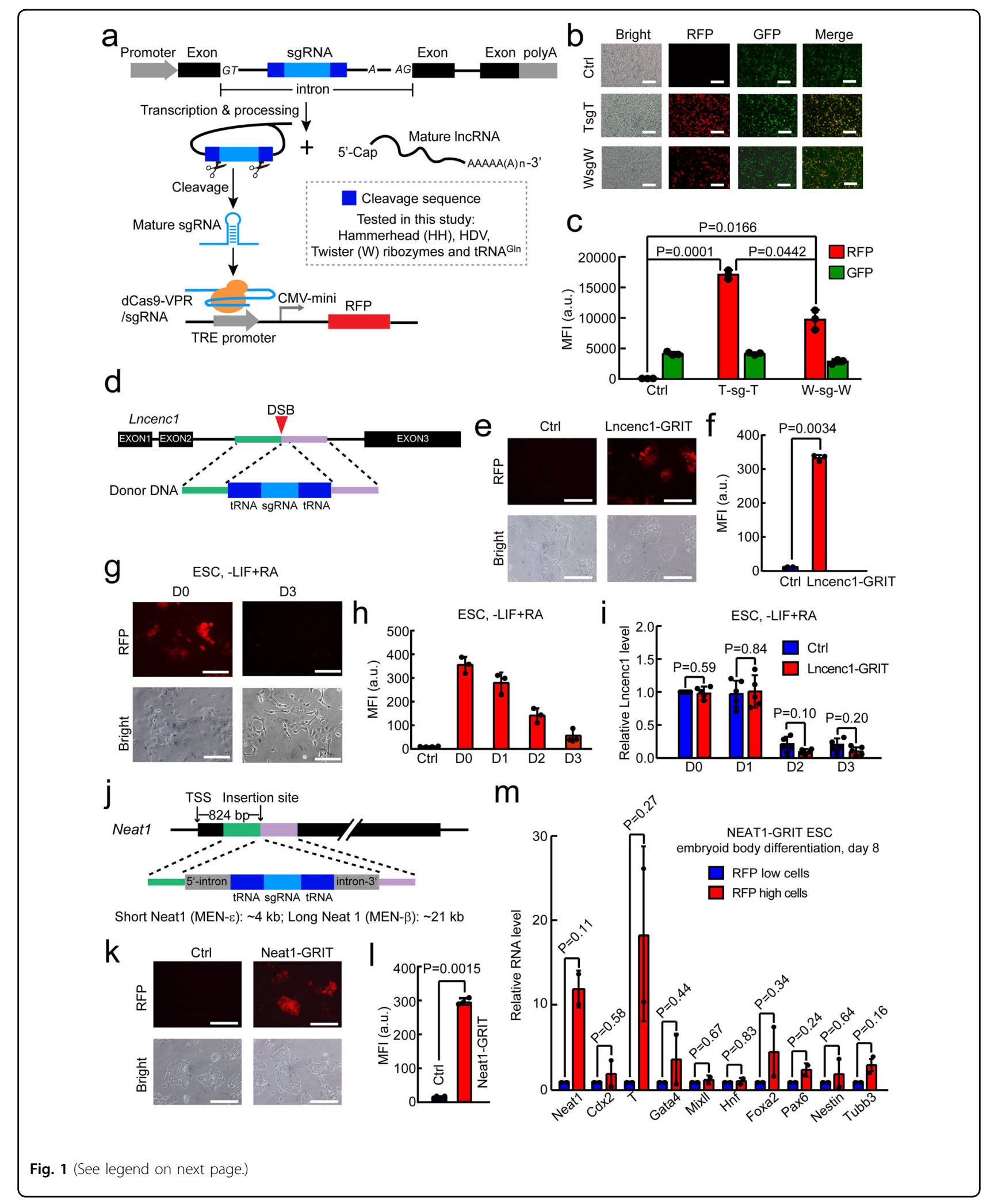


(see figure on previous page)

Fig. 1 GRIT monitors the promoter activity of IncRNAs and stem cell differentiation. a Schematic design of GRIT reporter system. GRIT cassette refers to pre-sgRNAs located in an intron. After the transcription of host gene, removal of flanking RNA cleavage sequences leads to the maturation of sgRNA, which activates the downstream CRISPR-on reporter system. Hammerhead, HDV and Twister ribozyme sequences are in Supplementary Table S1. b Representative microscopy images showing RFP and GFP expression in HEK293T transfected with dCas9-VPR, TRE3G-RFP, and GRIT-GFP plasmids. For control, GFP plasmid without any sgRNA cassette in the intron was transfected with dCas9-VPR and TRE3G-RFP plasmids. The schematic for the design of this experiment is shown in Supplementary Fig. S1a. Scale bars, $200 \mu \mathrm{m}$. The experiments were repeated three times independently with similar results. TsgT, tRNA-flanked sgRNA. WsgW, Twister ribozyme-flanked sgRNA. c Quantification of mean RFP and GFP intensity of $\mathbf{b}$. Shown are mean $\pm \mathrm{SD}, n=3$ independent experiments. The P-value was calculated by one-way ANOVA with two-tailed Tukey's multiple comparisons test. d Schematic of GRIT knock-in strategy for Lncenc1. After the establishment of dCas9-VPR and TRE3G-RFP transgenic mouse ESCs, the TsgT element is knocked in the second intron of Lncencl locus through CRISPR-Cpf1-assisted homologous recombination. e Representative images showing RFP expression in Lncenc1-GRIT ESCs. Scale bar, $200 \mu \mathrm{m}$. f Mean RFP intensity of Lncenc1-GRIT ESCs and control ESCs. Shown are mean \pm SD, $n=3$ independent experiments. The $P$-value was calculated using two-tailed unpaired Student's $t$-test. $\mathbf{g}$ Representative images showing RFP expression in undifferentiated and differentiated Lncenc1-GRIT ESCs. Scale bar, $200 \mu \mathrm{m}$. $\mathbf{h}$ Quantification of mean RFP intensity during the continuous differentiation process of Lncenc1-GRIT mESCs. Shown are mean \pm SD, $n=3$ independent experiments. i RT-qPCR analysis of Lncenc1 expression during the differentiation process of Lncenc1-GRIT and control ESCs. Shown are mean \pm SD, $n=5$ independent experiments. The $P$-value was calculated using two-tailed paired Student's t-test. $\mathbf{j}$ Schematic of GRIT knock-in strategy for Neat 1 locus. An artificial intron based on the second intron of human RPL18a gene containing GRIT elements was knocked into the Neat1 locus. $\mathbf{k}$ Representative images showing RFP expression in Neat1-GRIT cells. Scale bar, $200 \mu \mathrm{m}$. I Mean RFP intensity of Neat1-GRIT ESCs. Shown are mean \pm SD, $n=3$ independent experiments. The $P$-value was calculated using two-tailed unpaired Student's t-test. $\mathbf{m}$ RT-qPCR analysis of various differentiation markers in RFP high and low cells from day 8 differentiating embryoid bodies of Neat1-GRIT ESCs. EB, embryoid body. Shown are mean \pm SD, $n=2$ independent experiments. The $P$-value was calculated using two-tailed paired Student's t-test. Control ESCs for Lncenc1-GRIT or Neat1-GRIT were ESCs with dCas9-VPR and TRE3G-RFP transgenically integrated but without knock-in of TsgT cassette.

Furthermore, by comparing the expression of Lncenc1 and Oct4 during the differentiation process of control and Lncenc1-GRIT ESCs (Fig. 1i; Supplementary Fig. S2d), we concluded that knock-in of tRNA-flanked sgRNA did not impact the differentiation potential of mouse ESCs. To check whether RFP level may reflect the stages of differentiation, we sorted out high and low RFP populations in day 2 differentiated Lncenc1-GRIT cells and analyzed the expression of pluripotency genes (Supplementary Fig. S3a). Interestingly, Oct4, Nanog, and Sox2 were indeed higher in RFP high cells than in RFP low cells (Supplementary Fig. $\mathrm{S} 3 \mathrm{~b})$. These results demonstrate the potential of GRIT to report the promoter activity of lncRNAs and as an indicator to monitor the differentiation status of stem cells.

NEAT1 is a lncRNA serving as a structural organizer of paraspeckle and has been shown to play important roles from gene regulation to cancer progression ${ }^{6}$. In addition, NEAT1 is an intronless gene. We constructed NEAT1GRIT ESCs by inserting a mini-intron containing tRNAflanked sgRNA (Fig. 1j). As expected, RFP was significantly induced in NEAT1-GRIT ESCs (Fig. 1k, l). In addition, the insertion of mini-intron did not affect the expression of NEAT1 RNA and pluripotency genes including Oct4, Nanog, Sox2, and Klf4 (Supplementary Fig. S4a). We then performed embryoid body differentiation of NEAT1-GRIT ESCs and sorted out RFP high cells at day 8 (Supplementary Fig. S4b). As expected, qRTPCR analysis showed that RFP high cells express higher level of NEAT1 (Fig. 1m). In addition, mesoderm marker $\mathrm{T}$ brachyurary was highly upregulated in RFP high cells (Fig. 1m), indicating that high NEAT1 expression may mark certain cell lineages from mesoderm. Finally, we made a GRIT reporter for H19 lncRNA to drive TRE-GFP expression (Supplementary Fig. S5a). Consistently, GFP expression was highly induced in H19-GRIT ESCs (Supplementary Fig. S5b) and the knock-in of GRIT did not affect the expression of H19 lncRNA or pluripotency genes (Supplementary Fig. S5c). Together, these data demonstrate the generality of GRIT to serve as a reporter for monitoring the promoter activity of noncoding RNAs.

In summary, we constructed a lncRNA reporter GRIT with CRISPR-on system by insertion of a tRNA-flanked sgRNA in endogenous lncRNA loci. We showed that GRIT is useful for tracking ESC differentiation and labelling specific cell lineages. A recent study from Gao et al. reported a similar design named as Ents ${ }^{7}$. Ents uses ScFV-P65-HSF1 instead of VPR to activate gene expression and optimized mini-CMV-mCherry as a reporter. Different from traditional methods to report promoter activity by knocking in a protein such as GFP or luciferase, both GRIT and Ents strategies knock-in a smaller DNA fragment that will not change the noncoding status of lncRNAs. In Ents strategy, the authors knocked in the sgRNA cassette downstream of the polyA site. Here we knocked in the sgRNA cassette in the middle of ncRNA sequences. While the Ents strategy may have less impact than the GRIT strategy on the expression or function of monitored genes, the faithfulness of Ents in monitoring the promoter activity could be reduced in certain cases since the cleavage at the polyA site may affect the stability of downstream transcript. In the case of Lncenc1 in mouse ESCs, Ents strategy achieved $\sim 3$-fold induction of mCherry expression, while the GRIT strategy in this study achieved $\sim 35$-fold induction of RFP expression. However, 
the exact effectiveness of two strategies can only be concluded when both strategies are compared in the same system side by side. In addition, for both Ents and GRIT, the expression of dCas9 or associated proteins could affect their faithfulness in monitoring the promoter activity. This issue may be addressed by knocking the expression cassette of dCas9 and associated proteins into constitutively active gene loci. Finally, when sgRNAs are designed to target endogenous DNA locus, GRIT may be utilized to edit or manipulate the expression of endogenous genes. We expect that GRIT will be applicable in uncovering molecular mechanisms regulating the transcription of lncRNAs, tracking cell fate switch during differentiation, reprogramming or disease progression and integrating the promoter activity for synthetic biology applications.

\section{Acknowledgements}

We would like to thank Drs Lu-Feng Hu, Jing Hao, and other members of Wang laboratory for discussion of the project. We thank the Flow Cytometry Core at National Center for Protein Sciences at Peking University, particularly Huan Yang, Jia Luo, and Dr. Hongxia Lv for technical help. This study was supported by The National Key Research and Development Program of Ministry of Science and Technology of the People's Republic of China (2016YFA0100701 and 2018YFA0107601) and National Natural Science Foundation of China (91940302, 31821091, and 32025007) to Y.W.

\section{Author contributions}

Y.-T.Z. performed all the experiments. Y.-T.Z. and Y.W. interpreted the data. Y.W. conceived and supervised the project and wrote the manuscript.
Conflict of interest

The authors declare no competing interests.

\section{Publisher's note}

Springer Nature remains neutral with regard to jurisdictional claims in published maps and institutional affiliations.

Supplementary information The online version contains supplementary material available at https://doi.org/10.1038/s41421-021-00272-3.

Received: 9 January 2021 Accepted: 14 April 2021

Published online: 15 June 2021

\section{References}

1. Djebali, S. et al. Landscape of transcription in human cells. Nature 489, 101-108 (2012).

2. Kopp, F. \& Mendell, J. T. Functional classification and experimental dissection of long noncoding RNAs. Cell 172, 393-407 (2018).

3. Wang, X. W. et al. A microRNA-inducible CRISPR-Cas9 platform serves as a microRNA sensor and cell-type-specific genome regulation tool. Nat. Cell Biol. 21, 522-530 (2019).

4. He, X. et al. Boosting activity of high-fidelity CRISPR/Cas9 variants using a tRNA(Gln)-processing system in human cells. J. Biol. Chem. 294, 9308-9315 (2019).

5. Sun, Z. et al. The long noncoding RNA Lncenc1 maintains naive states of mouse ESCs by promoting the glycolysis pathway. Stem Cell Rep. 11, 741-755 (2018).

6. Wang, Y. \& Chen, L. L. Organization and function of paraspeckles. Essays Biochem. 64, 875-882 (2020).

7. Gao, N. et al. Endogenous promoter-driven sgRNA for monitoring the expression of low-abundance transcripts and IncRNAs. Nat. Cell Biol. 23, 99-108 (2021). 